\title{
Regímenes de bienestar y roles familiares: un análisis del caso español
}

\author{
Ana M. Guillén \\ U niversidad de 0 viedo \\ O viedo. Spain
}

\section{Resumen}

El texto plantea la influencia de la incorporación de la mujer al mercado de trabajo y la existencia del estado del bienestar en los roles familiares.

Palabras clave: roles familiares, mercado de trabajo, política social familiar.

Abstract. Welfare State and family roles: analysis of the Spanish case

The article shows the influence of woman's incorporation into the labour market and the existence of a Welfare State in family roles.

Key words: family roles, labour market, family social policy.

\section{Sumario}

1. ¿Continuidad o cambio en los roles familiares?

2. Estado del bienestar, mercado de trabajo y familia

3. Política social para la familia y servicios sociales
4. Sistema de garantía de ingresos y mercado de trabajo

5. Conclusiones

Bibliografía

\section{1. ¿C ontinuidad o cambio en los roles familiares?}

En España, las mujeres se están incorporando al mercado de trabajo en proporciones crecientes y durante períodos de tiempo más largos, así como también están surgiendo nuevas formas de convivencia, procesos ambos que se pueden considerar como generadores de nuevas identidades de rol. En efecto, el trabajo de la mujer fuera de casa trae consigo el surgimiento de un nuevo modo de articulación entre las estructuras familiares y los sistemas de producción, y puede suponer un cuestionamiento de la especificidad de los roles 
en el seno de la pareja, así como cambios en la gestión del tiempo y del patrimonio familiar. Tal como indica Garrido M edina (1993: 174), «cuando se obtienen recursos en el mercado disminuye la propensión a proporcionar servicios domésticos gratuitos por dos vías. Por una parte, se dispone de menos tiempo para ello y, por otra, aumenta la probabilidad de rentabilizar la compra de esos servicios en el mercado». Sin embargo, la evidencia muestra que la división tradicional de funciones dentro de la familia sigue permaneciendo en gran medida, tal como indican las encuestas de uso del tiempo dentro del hogar.

Los estudios publicados por el Instituto de la M ujer indican que, en general, son las mujeres las encargadas de las tareas del hogar, a gran distancia de los hombres. Los hombres casados parecen dedicar menos tiempo a las tareas domésticas que los varones en general, y lo contrario ocurre con las mujeres casadas, de lo que se deriva que las desigual dades se agravan con el matrimonio. Y, lo que es más importante, entre las mujeres trabajadoras, la actividad fuera del hogar reduce la dedicación a las tareas domésticas, pero la reducción es de escasa entidad y, además, la disminución del tiempo dedicado durante los días laborables se compensa con un aumento en los fines de semana. Por su parte los varones trabajadores también incrementan la participación y el tiempo invertido en el trabajo los sábados y domingos, pero menos que las mujeres, siendo la dedicación de éstos últimos siempre muy inferior a lo largo de toda la semana. Los varones pertenecientes a los niveles educativos más altos participan en mayor grado que los demás, pero no invierten más tiempo, por lo que el reparto del trabajo no se produce ni siquiera en los niveles educativos más altos. Por otra parte, hay que señalar que la distribución del trabajo es más asimétrica en el caso del trabajo doméstico en sentido restringido que en el tiempo dedicado a la adquisición de bienes y servicios y al cuidado de los niños, aunque las distancias entre ambos sexos son notorias en todas estas actividades (Ramos Torres, 1990).

Estos datos indican que la realización de una actividad remunerada fuera del hogar supone una menor dedicación a las tareas domésticas por parte de las mujeres, pero también que su participación en el mercado laboral no les permite olvidar sus responsabilidades domésticas. En pocas palabras, la menor dedicación en días de trabajo se compensa con el aumento de actividad los fines de semana y, probablemente, transfiriendo las tareas a otras manos que no parecen ser las de los hombres. Se produce, pues, una tímida modificación del rol femenino tradicional, pero no del masculino.

U na de las posibles causas de la persistente división de roles está en las oportunidades que brinda a las mujeres el mercado de trabajo en comparación con otros países de nuestro entorno, tales como menores oportunidades de ocupar puestos de responsabilidad, la relativa escasez de contratos a tiempo parcial, los bajos sal arios y la incidencia de las ayudas familiares. Las conclusiones que ofrece el estudio Actividad laboral de la mujer en relación a la fecundidad (Femández M éndez de Andrés, 1987: 121-122), centrado en el caso español, son clarificadoras de los motivos por los que las mujeres casadas se animan a participar en el mercado de trabajo. La decisión de trabajar fuera del hogar se 
explica fundamentalmente por el coste oportunidad de quedarse en casa. La incorporación al mercado de trabajo de las mujeres casadas está relacionada con los aumentos de salario potencial en el mercado y, de forma menos intensa, con el número de hijos y con el nivel salarial del cónyuge. El número de hijos constituye un desincentivo porque incrementa la productividad del tiempo en el hogar, mientras que el nivel salarial del cónyuge está relacionado inversamente con la actividad debido a un reparto de tareas conducente a lograr maximizar la utilidad conjunta. Por otra parte, el nivel de educación resulta crucial - mucho más quela edad o el estado civil- para determinar el tipo de ocupación que desempeñan las mujeres. Los mayores niveles de educación, junto con la posibilidad de obtener salarios más elevados, explican el acentuado descenso en la tasa de fecundidad y son responsables principales de la incorporación de las mujeres al mercado de trabajo.

D esde luego, no cabe olvidar que la influencia de las oportunidades que ofrece el mercado de trabajo es sólo parte de la cuestión. El cambio de valores y actitudes frente al trabajo de la mujer y la puesta en práctica de un proceso de socialización de los géneros más igualitario pudiera ser mucho más importante como motor de modificación de los roles tradicionales. D e hecho, a pesar de los incentivos que suponen el mayor nivel de educación y la posibilidad de obtener salarios más altos, actitudes sociales tales como las de la familia, del marido, del empleador ante el trabajo de la mujer casada y soltera, pueden restringir el acceso de las mujeres al empleo y, con ello, ayudar a perpetuar los roles familiares tradicionales. Las mujeres tienen mucha menos familiaridad que los hombres con la regulación del mercado de trabajo, con las prácticas sindicales, y son por ello mucho más proclives a aceptar condiciones de trabajo menos favorables. Además, el trabajo de la mujer se sigue concibiendo en muchos casos como una ayuda, como un complemento al tra bajo principal. Esta posición la comparten muchos maridos, familiares y empleadores, lo cual no puede dejar de influir sobre las mujeres cuando deciden salir de casa a buscar trabajo. Incluso las propias mujeres consideran lo mismo en muchos casos.

A pesar de que ambos sexos han alcanzado niveles similares de educación en la última década en España, hombres y mujeres reciben una educación muy distinta dentro de la familia. D e hecho, a las mujeres se les enseña cómo realizar las tareas del hogar y se las implica activamente en ellas a diario, mientras que a los hombres se les mantiene al margen en una proporción mucho más alta. Además, puesto que los hijos permanecen en el hogar en España hasta edades avanzadas, esta situación de división de tareas se perpetúa durante mucho más tiempo que en otros países y son, normalmente, las recién casadas las encargadas de enseñar a sus maridos el trabajo doméstico, con el agravante de tener que forzarlos o convencerlos en muchas ocasiones de la conveniencia de que compartan unas tareas que no han realizado hasta entonces y sobre las que tienen una visión negativa. Asimismo, en otros países, las altas tasas de divorcio ponen a los hombres en la situación de tener que ocuparse de realizar tareas domésticas, pero en España, la escasa incidencia del fenó- 
meno en términos comparativos hace que esta situación tenga mucho menos impacto. Además, son las mujeres las que normalmente mantienen la custodia de los hijos (Valiente, 1995c: 8).

Pero, además, existe otro modo de mirar hacia las razones que pueden inducir a las mujeres a buscar trabajo fuera del hogar y con ello producir una modificación, por muy limitada que sea, de su rol tradicional. M e refiero a las alternativas que existen, ya sean públicas o privadas, de ser sustituidas (al menos parcialmente) en tareas tradicionalmente asignadas a las mujeres, tales como el cuidado de los hijos o de los ancianos. La provisión pública de este tipo de servicios forma parte del estado del bienestar, en concreto del nivel de oferta de servicios sociales personales. Aquellas mujeres que tienen que dedicar gran parte de su tiempo a dichas actividades y que no encuentran una forma de ponerlas en otras manos que no sea muy costosa desde el punto de vista económico, tenderán a percibir el coste oportunidad de trabajar fuera del hogar como muy alto.

Es por el lo que este artículo examina de forma comparada con otros países de la Unión Europea la configuración del sistema de bienestar español, y en particular considera la evolución de los servicios sociales durante la última década en España y de las prestaciones o subsidios familiares. Asimismo, se tiene en cuenta el sistema de garantía de ingresos, porque la forma en que esté organizado puede tener consecuencias para las decisiones que toman las familias en cuanto al reparto de trabajo fuera y dentro del hogar. Lo que se busca es llamar la atención sobre el hecho de que los modos como se construye un Estado del bienestar y las formas de interacción entre la política social y el mercado de trabajo pueden influir sobre la reproducción de roles familiares y las posibilidades de producción de nuevos roles, al suponer bien sea incentivos 0 desincentivos a la búsqueda de empleo de las mujeres fuera del hogar y a la permanencia en el mercado de trabajo.

Las familias españolas actúan en muchos casos como proveedoras directas de servicios sociales, como «agencias de empleo» a través de sus relaciones informales y se hacen cargo del mantenimiento de muchos desempleados. $\mathrm{N}$ o deja de resultar paradójico que en estas circunstancias la política social para la familia se encuentre tan poco desarrollada en España. Parte de la explicación a esta situación aparentemente contradictoria se puede encontrar en las relaciones que se han ido perfilando entre las políticas sociales, el mercado de trabajo y la institución familiar.

\section{Estado del bienestar, mercado de trabajo y familia}

Esping-Andersen (1990) defiende que existen tres tipos de regímenes de bienestar $^{1}$ en el mundo occidental. D ejando a un lado el caso de los Estados

1. La obra de Esping-Andersen (1990) trata de entender las políticas sociales no como un conjunto de instituciones y programas aislados, sino dentro del ámbito más amplio de la 
Unidos, ejemplo del régimen «iberal», en Europa se puede hablar de dos tipos fundamentales de regímenes de bienestar. El primero de ellos, es el Ilamado coloquialmente «modelo escandinavo» o régimen socialdemócrata, y estaría basado en el concepto de universalización y armonización entre las clases sociales y entre los géneros. En estos países, el desarrollo del estado del bienestar - la universalización de las prestaciones- permitió a las mujeres lograr un empleo, a la vez que se creaba un mercado de trabajo para las mujeres. Pero las propias virtudes del modelo condujeron a consecuencias no deseadas y negativas para el colectivo femenino, ya que, a la larga lo que se produjo fue la segmentación del mercado de trabajo por género: las mujeres trabajan sobre todo en el sector servicios dependiente de las prestaciones sociales públicas, mientras que los hombres lo hacen en el sector privado.

España - junto con Alemania, Francia, Italia, Bél gica, Austria, Portugal y en cierta medida $\mathrm{H}$ olanda - pertenecería al grupo de países que han construido su Estado del bienestar a través de sistemas de seguridad social profesionalistas. Una de las características de este modelo, bautizado como «conservador» por Esping-Andersen, es la de su estructuración corporativista, es decir, la de la existencia de una segregación de estatus, que se refleja en la presencia de un número el evado de regímenes especiales que diferencian categorías laborales, acompañado de un tratamiento favorable de los empleados públicos. En esencia, dicho autor considera que este tipo de régimen de bienestar se sustenta, fundamentalmente, en dos pilares; por un lado, en un concepto tradicional de la familia, de inspiración católica, por el cual el núcleo familiar se constituye en una fuente de provisión de cuidados para cada uno de sus miembros, especialmente aquéllos menos válidos (niños, ancianos, enfermos); por otro lado, en la consideración del trabajador masculino perteneciente al núcleo familiar como el garante, mediante su salario, de la cobertura de las necesidades familiares. Este esquema, en el que el hombre no sólo contribuye con su trabajo a la cobertura de sus necesidades individuales, sino también a las de su familia, está ligado a la existencia de un grado el evado de seguridad en el trabajo y de la necesidad de articular medidas que garanticen la capacidad del trabajador de seguir cubriendo las necesidades familiares en aquellos momentos en los que, por motivos biológicos o de otra índole, aconteciese al guna interrupción en su actividad laboral. Algunas de las consecuencias más destacadas o rel evantes de este esquema serán la rigidez del mercado laboral, con un al to porcentaje de empleo fijo y la pervivencia de la familia como proveedora de prestaciones sociales, circunstancia esta que provoca un fortalecimiento de los lazos que ligan a la mujer a las labores propias

economía política, es decir, de la actividad del Estado en la gestión y en la organización de la economía nacional. En consecuencia, la atención se centra en las relaciones entre la política social de un determinado país y el mercado de trabajo, la distribución salarial, la estra tificación social y las decisiones macroeconómicas. D e ahí que acuñe el término de «capitalismo del bienestar» para bautizar a todo ese complejo, y el de «regímenes de bienestar» para referirse a las modalidades particulares (nacionales) del anterior. 
del ámbito del hogar, retrasando y dificultando su incorporación al mercado laboral.

En una línea muy similar de argumentación, Van Kersbergen (1995) considera que el principio de subsidiariedad es el que guía la intervención del Estado en política social en los países católicos: el Estado no tiene obligación de tratar a todos por igual sino de trabajar a través de los grupos sociales existentes. De esos grupos sociales, la familia tiene primacía, es la unidad básica de la sociedad (no el individuo). La igualdad tampoco es el objetivo de la vida familiar, sino el logro de una unidad orgánica y la división del trabajo entre los géneros bajo la dirección del marido. El concepto de ciudadanía no es el básico en este régimen de bienestar, puesto que se trata de un concepto asociado a los individuos. Además, el principio de subsidiariedad supone también la resistencia a transferir poder al Estado. D e ahí que los modelos de seguridad social en los países de tradición católica se financien principalmente a través de las contribuciones de empresarios y trabajadores y no a través de impuestos. En estas circunstancias no es sorprendente que los resultados de la política social afecten de forma distinta a los géneros.

\section{Política social para la familia y servicios sociales}

Si aceptamos que la configuración del sistema de previsión social afecta de forma distinta a hombres y mujeres, cabe entonces deducir que aquellos sistemas que mantengan a las mujeres en el hogar tenderán a ayudar a reproducir los roles tradicionales. ¿Cuál es exactamente esa configuración en España? Se trata de dilucidar hasta qué punto el sistema de bienestar ofrece oportunidades a las mujeres para salir a trabajar y conjugar el empleo con la vida doméstica y la crianza de los hijos. En este sentido, hay que referirse al menos al grado de desarrollo de las políticas sociales para la familia, es decir, a las prestaciones y subsidios de que puede disponer una familia, y al grado de desarrollo de los servicios sociales personales, es decir, a la oferta pública de cuidados a los niños de corta edad, minusválidos y ancianos.

Resulta difícil establecer una definición de lo que es la política social para la familia porque el concepto ha variado históricamente y porque los objetivos que persigue son muchas veces contradictorios entre si: potenciación de la fecundidad, reducción de la pobreza infantil, reforzamiento del papel de las mujeres como madres solamente o como trabajadoras y madres a la vez. En general, cabe defender que la política social para la familia debe comprender, en primer lugar, la regulación de los derechos y deberes dentro de la familia, así como entre la familia y el resto de las instituciones sociales y políticas, y, en segundo lugar, la oferta de servicios y prestaciones económicas (Rodríguez Cabrero, 1994). Es un hecho bien documentado que la política social para la familia en su vertiente de oferta pública de transferencias y servicios está escasamente desarrollada en España (I glesias de U ssel y M eil, 1994; Valiente, 1995b). En España no existe ni siquiera un departamento administrativo espe cializado en ella. Las prestaciones familiares han pasado de constituir el 17\% 
del gasto total en prestaciones económicas en 1975 al 1,17\% en 1990 (Velarde, 1990: 164). Parece que como reacción al familismo paternalista del régimen anterior se ha pasado a una situación si no hostil, sí de clara indiferencia hacia la familia, razón ésta, por otro lado, que explica mejor la falta de atención a la familia por parte de los poderes públicos y de partidos y grupos de interés que la pretendida pervivencia de la ideología católico-social en España, tal como lo interpreta Esping-Andersen (1994).

De la falta de atención pública a la familia es indicativo que las prestaciones familiares fueron reformadas en 1990 por primera vez desde los inicios de la transición a la democracia. La cuantía de los beneficios que eran per cibidos con anterioridad se puede etiquetar de residual, ya que a principios de los años setenta era ya baja y nunca se actualizaron desde entonces, de forma que en 1990 las cantidades percibidas eran testimoniales². D esde la reforma del noventa, las prestaciones familiares se han incrementado, son iguales para contribuyentes y no contribuyentes, y dependen del nivel salarial y de las circunstancias personales (sobre todo del hecho de la circunstancia de tener un minusválido a cargo). A pesar de que uno de los objetivos de la reforma consistía en incentivar el trabajo de las mujeres fuera del hogar y permitir a los hombres disfrutar del permiso de maternidad sin perder derechos contributivos, el nivel salarial máximo para poder disfrutar de los beneficios es muy bajo: un millón de pese tas al año, lo que supone una cantidad sólo ligeramente superior al sal ario mínimo interprofesional. Esta circunstancia ha tenido como consecuencia la acentuación del carácter asistencial del sistema (Rodríguez Cabrero, 1994). Las políticas familiares también incluyen detracciones en los impuestos por cada descendiente dependiente, así como descuentos en el transporte, la educación y créditos para familias numerosas ${ }^{3}$.

La comparación de los subsidios familiares con otros países de la Unión Europea nos indica que en 1991 en España los subsidios familiares por joven menor de veinte años representaba solamente el 0,5\% del PIB por habitante, es decir, se trataba del país que menos dedicaba a esta prestación, cantidad treinta veces menor que la del país que más gastaba (D inamarca). En términos del porcentaje sobre el sal ario medio neto, los subsidios familiares para unidades con un hijo suponen en España el 2\%, sólo por encima de Francia con el $1 \%$. Sin embargo, mientras estos porcentajes aumentan mucho en Francia para las familias con dos o más hijos, en España y en el resto de los países del sur de Europa permanecen muy bajos, sobre todo en el caso de España e Italia (Comisión Europea, 1994: 50, 61). Además de las prestaciones familiares propiamente dichas, hay que tener en cuenta los subsidios de desempleo asisten-

2. En concreto consistían en 250 pesetas al mes por hijo a cargo y en otra asignación de 375 pesetas al mes por cónjuge dependiente. Esta última se suprimió en 1985. Los asegurados también podían percibir seis mil pesetas por matrimonio y tres mil por el nacimiento de cada hijo, en ambos casos como pago único.

3. D esde noviembre de 1995, las familias con tres hijos pueden acceder a los beneficios por familia numerosa. 
cial para parados con hijos a cargo, los salarios sociales que son proporcionales al tamaño de la familia, y las pensiones no contributivas que priorizan a los ancianos y minusválidos que viven en familia. Aún aś, se puede afirmar que las prestaciones familiares son muy escasas en términos comparativos.

El conjunto de medidas que protegen la maternidad de las mujeres trabajadoras puede desglosarse en España en dos grandes elementos: por una parte, el derecho de la mujer empleada a ausentarse del trabajo por el nacimiento, la adopción o el acogimiento previo de un hijo, con la garantía de conservar su puesto, su antigüedad y sus derechos a tener una pensión de jubilación; por otra parte, el derecho a recibir una prestación económica pagada durante ese permiso. H asta diciembre de 1994, fecha en la que se modificaron los programas de protección a la maternidad en España, la situación derivada de la maternidad de la mujer trabajadora se venía asimilando a la incapacidad laboral por enfermedad común, por lo que su tratamiento normativo a efectos de protección social era semejante al de esta contingencia. H oy día, pues, la situación de maternidad se encuentra regulada separada de la incapacidad temporal, con el resultado de no asimilarse a una enfermedad.

Además, y en consonancia con este cambio de concepto, en diciembre de 1994 la cuantía de la prestación económica se elevó desde el 75\% del salario (base reguladora) al 100\%. También entre las modificaciones que sufrió la prestación por maternidad se encuentra la de que la condición exigida para disfrutar de la prestación es la acreditación de ciento ochenta días de cotización dentro de los cinco años inmediatamente anteriores al parto, condición más laxa que la que estaba en vigencia anteriormente y que exigía este requisito durante el año anterior al parto. D esde 1989, la duración del descanso es de dieciséis semanas ininterrumpidas, ampliables a dieciocho en caso de parto múltiple. Este período se distribuye como desee la interesada, con la única salvedad de que seis semanas se disfruten después del parto. El padre puede disfrutar, alternativamente, de cuatro de las últimas semanas del permiso. D esgraciadamente, no existen datos que permitan saber cuántos hombres han decidido acogerse a este derecho de este permiso en vez de sus esposas trabajadoras, pues podría ser un indicador de cambio en la distribución de las responsabilidades familiares. Sin embargo, cabe suponer que dicha cantidad sea muy escasa.

0 tro de los cambios recientes en la protección de la maternidad ha sido la adopción de nuevas normas que tratan de garantizar la continuidad de la vida laboral de las mujeres y los hombres trabajadores con hijos a cargo. En marzo de 1995, se ha aprobado una nueva regulación que se denomina «permiso parental y por maternidad», que mantiene el derecho de los trabajadores a un período de excedencia no superior a tres años, para atender al cuidado de cada hijo, pero que lo complementa permitiendo que sea computado a efectos de antigüedad. Además, el trabajador tendrá derecho a la reserva de su puesto de trabajo durante el primer año de excedencia, y durante los dos restantes la reserva quedará referida a un puesto de trabajo del mismo grupo profesional o categoría equivalente. Esta medida, al menos en teoría, favorecerá la continuidad de las carreras profesionales de las mujeres, aunque cabe preguntarse 
cuantas empresas privadas estarán dispuestas a ponerla en práctica. En general, todas estas modificaciones en la protección por maternidad han situado a España a un nivel muy parecido al del resto de los países de la Unión Europea, o incluso más generoso en al gunas cuestiones como, por ejemplo, la cuantía de la prestación por maternidad con respecto al salario antes percibido (Comisión Europea, 1994).

L as prestaciones familiares y por maternidad ayudan a que aquellas mujeres que ya están trabajando tengan más facilidades para conciliar su trabajo fuera y dentro del hogar sin que su carrera profesional sufra interrupciones. Pero tanto para las mujeres trabajadoras como para las que están buscando empleo, el grado de desarrollo de los servicios sociales personales es importante, puesto que la oferta pública las libera de tareas tradicionalmente femeninas. La oferta de servicios sociales está dividida en estos momentos aproximadamente al cincuenta por ciento entre el sector público y el sector privado (mercantil y voluntario). Éste último ha crecido considerablemente en los últimos años debido, entre otras razones, a la escasez de oferta pública y al crecimiento de la demanda. Los poderes públicos siempre han considerado al sector privado como subordinado y subsidiario del público, pero, sin embargo, el sector mercantil carece aún de una inspección y control adecuados (Casado, 1994).

L os servicios sociales públicos incluyen en España programas muy diversos, entre los que se encuentran la asistencia a la tercera edad (asistencia domiciliaria, residencias, vacaciones), a los minusválidos (atención especializada, programas de integración social y laboral), a las minorías étnicas, a los niños, a los jóvenes, a las mujeres y a los drogadictos, así como también las prestaciones no contributivas y las subvenciones a organizaciones de ayuda no guberna mentales. Este área de la política social se financia a través de impuestos, en parte a través de las comunidades autónomas y en parte a través de transferencias del M inisterio de Asuntos Sociales, y a través de las cotizaciones de los beneficiarios de la Seguridad Social. Ante la imposibilidad de ofrecer aquí un análisis de todas las facetas de los servicios sociales, voy a ocuparme de los destinados a la tercera edad, a los minusválidos y a los niños.

Por lo que respecta a los servicios sociales para minusválidos, la oferta en España incluye programas de prestaciones económicas, de acceso a la información, de fomento del empleo, medidas para la eliminación de barreras físicas, prestación de servicios de atención domiciliaria y fomento de la asistencia sanitaria por los organismos públicos. El marco legal que regula todo lo concerniente a los discapacitados, deficientes y minusválidos, la Ley de Integración del M inusválido, aprobada en 1982, supuso un importante intento de adecuar la normativa española a la de nuestro entorno, así como también de ofrecer un abanico muy amplio de medidas que posibilitaran la integración social de este colectivo, colectivo que ascendía al 15\% de la población en 1985 (IN E, 1986). Q uizás esta amplitud ha tenido la consecuencia de que algunos aspectos importantes, tales como los programas de rehabilitación y prevención, han recibido muy poca atención y en otros casos han resultado de escasa eficacia, 
como, por ejemplo, en el fomento del empleo. Es posible que esta situación también se deba al hecho de que los servicios demandados con más intensidad son claramente las prestaciones económicas, que han acaparado la atención y los recursos de la Administración en el período transcurrido desde la aprobación de la LISM I (Casado, 1992).

La comparación global entre los países de la U nión de los sistemas de protección a los minusválidos presenta muchas dificultades, debido a la gran diversidad de programas y de condiciones de acceso a los mismos existentes. Todo ello se complica aún más si tenemos en cuenta que, tal como ocurre en parte en España, muchas de las prestaciones se regulan, se organizan y se gestionan a nivel local, lo que a veces introduce diferencias importantes difíciles de generalizar. A pesar de todos estos problemas, de los estudios comparativos se puede deducir que el nivel de protección y de desarrollo de los programas sociales para minusválidos, aunque se ha incrementado mucho durante los últimos años en los países del sur de Europa, todavía se encuentra a cierta distancia del logrado en el resto de los países de la U nión (M inisterio de Asuntos Sociales, 1989).

Entre los servicios que se ofertan a la tercera edad se encuentran en España la asistencia médica especializada y preventiva, las residencias y la ayuda para la vivienda, la atención domiciliaria, los clubes y hogares de jubilados, las vacaciones organizadas, y el apoyo económico a los familiares con ancianos. H oy día existen casi 110.000 plazas residenciales (públicas y privadas), lo que supone una ratio del $2 \%$, que se encuentra todavía a distancia del 3,5\% recomendado en los países mediterráneos o del $4,5 \%$ de los países del centro y del norte de Europa. Los servicios de estancias diurnas en residencias fueron usados por 480 personas en 1992. El número de hogares y clubes para la tercera edad, también Ilamados centros de día, ascendía en 1992 a 2.000, sumando la iniciativa pública con la privada, distribuidos de forma muy irregular dentro del territorio nacional. En el mismo año, las personas que recibieron atención domiciliaria fue de casi 25.000. En general, resulta difícil establecer cuanto se aleja la oferta de servicios para las personas mayores de la demanda, pero la escasa cuantía de las cifras aquí expuestas son indicativas de que la distancia tiene que ser grande. Los ancianos españoles consideran las ayudas a domicilio como el segundo servicio más necesario, después de los servicios médicos especializados (M inisterio de Asuntos Sociales, 1993).

El análisis comparativo de las prestaciones para la vejez en Europa indica, como ocurría en el caso de la minusvalía, que los países miembros de la Unión presentan disparidades muy marcadas. La tendencia general parece conducir a la privatización de muchos de estos servicios en toda Europa. Sin embargo, si tenemos en cuenta de forma conjunta la oferta pública y la privada, los países del sur de Europa presentan desarrollos inferiores, tanto en la oferta de al ojamiento de larga estancia como en los servicios de atención a domicilio y centros de día (Guillemard, 1993).

En resumen, en lo relativo a los servicios sociales personales, la comparación con los países del norte y centro de Europa es clara: en España y en el resto de los países del sur esta faceta de la política social está menos desarro- 
$\| l a d a^{4} . \mathrm{H}$ ay que resaltar aquí que el estado del bienestar en España, tal como hemos visto, ofrece pocas ayudas a las familias y a las mujeres españolas, pero les pide mucho a cambio. En primer lugar, muchas familias españolas con hijos de corta edad no cuentan con la posibilidad de enviarlos a guarderías públicas. La oferta de plazas públicas se centra en España en la educación preescolar a partir de los tres años. En el curso 1991-1992 el porcentaje de niños que se ben eficiaron de esta oferta fue del $66,3 \%$ para los de cinco años; del $63,9 \%$ para los de cuatro, y del 17,6\% para los de tres. En comparación con la media de la Unión para los niños que son cuidados en centros públicos, estos porcentajes no son bajos, pues la cifra es del $65 \%$ para los niños de tres a seis años. Sin embargo, en el caso de los niños de dos años o menos, España presenta el porcentaje más bajo de toda Europa (1\%), incluso por debajo de los niveles de Grecia (4\%) y Portugal (6\%), y a considerable distancia del resto de los países (Valiente, 1995a).

Los mayores conviven con sus hijos o con otros familiares en proporciones elevadas: un $28,2 \%$ de los padres mayores de sesenta años y un $32,2 \%$ de las madres de la misma edad en 1991 (CES, 1995). Los minusválidos también son cuidados en grandes proporciones dentro de la familia. Incluso en el caso de los minusválidos psíquicos, la familia juega un papel crucial: el $84 \%$ de los enfermos psíquicos severos vive con su familia, una proporción muy alta si se compara con el $62 \%$ en Irlanda o el $21 \%$ en Suecia 5 . M uchas de estas familias declaran que no cuentan con suficiente ayuda social y financiera ni con la información necesaria para desarrollar su tarea adecuadamente. Los datos del último informeFO ESSA (1994: 1829) indican que casi el nueve por ciento de las familias españolas procuran atención en el propio hogar a personas dependientes, ya sean personas mayores, enfermos crónicos o hijos con discapacidades graves, y que este hecho es más frecuente entre las familias de menor nivel social.

En las situaciones de necesidad o de enfermedad, la mayoría de los españoles declaran que la familia constituyó la fuente de ayuda fundamental, con la excepción de casos graves de enfermedades psíquicas o de drogadicción en que también se menciona a algunas instituciones (Boletín CIRES, 1994). Incluso fuera del hogar, en las instituciones hospitalarias es extraño encontrar enfermos que no se encuentren acompañados por algún familiar día y noche. El número de familias extensas ha ido descendiendo de forma sostenida a lo largo de las dos últimas décadas, pero existe evidencia de la existencia de «familias amplias» formadas por varios grupos familiares que viven cada uno en su propio hogar y que mantienen relaciones más estrechas entre sí que con sus amigos o vecinos (N avarro, 1994). Alrededor del cuarenta por ciento de los hogares mantienen relaciones de ayuda familiar extendida: padres que ayudan a hijos

4. V éase, por ejemplo, el estudio editado por M unday (1993), que incluye a todos los países de la Unión Europea.

5. Según datos de una encuesta realizada por la Confederación Española de Agrupaciones Familiares de Enfermos M entales. V éase El País, 10 de marzo de 1995, p. 29. 
casados y nietos, hijos casados que viven en casa de los padres, hijos casados y emancipados que ayudan a padres mayores y padres mayores que viven en el hogar de un hijo (C asado, 1994: 1830).

Por otra parte, el número de jóvenes que permanecen en la familia es muy alto: un $77 \%$ de los jóvenes entre 18 y 29 años conviven con sus padres. Esta situación de postergación de la independencia incide en el incremento de cargas económicas que tienen que soportar las familias. Entre las razones que esgrimen los jóvenes para permanecer en la familia se encuentran la de que les gusta hacerlo así, pues les supone una convivencia más grata, pero también dicen necesitar que les mantengan (CIRES, 1994). A este fenómeno no es extraño, por supuesto, el escaso desarrollo de la política de vivienda, política social a fin de cuentas. U na vivienda de tipo medio costaba en 1991 más de cinco veces los ingresos anuales de una familia media, ingresos que los jóvenes no alcanzan ni remotamente cuando se incorporan al mercado de trabajo. Tampoco los alquileres resultan asequibles a los jóvenes, incluso los más bajos (Garrido M edina, 1993: 172).

0 tro aspecto de la evolución de las redes de parentesco está relacionada con la reaparición de un nuevo tipo de «familia extensa», compuesta por parejas casadas que viven con los padres de uno de sus componentes, es decir, parejas que han constituido su propia familia pero que no han establecido su propio hogar. En 1991, el 4,2\% de la población estaba en esta situación. Y lo que es más, el $20 \%$ de las parejas jóvenes que vivía en un hogar independiente declaraba depender económicamente de sus padres (CES, 1995).

Finalmente, el mercado de trabajo funciona (no estalla) y las altísimas tasas de paro se soportan en España gracias a que las familias comparten sus ingresos (procedentes de empleos seguros, precarios o de la economía sumergida o de las prestaciones sociales), acogiendo a parados y necesitados en general (Pérez D íaz y Rodríguez, 1994). Además, las familias españolas funcionan en muchos casos como oficinas de empleo, proporcionando a través de sus contactos un puesto de trabajo - sea del tipo que sea- a sus miembros.

En pocas palabras, las familias españolas cuidan, alimentan y dan cobijo a niños, ancianos, minusválidos, enfermos de distinta gravedad y parados, en muchos casos contando con servicios de apoyo muy escasos. La familia española realiza así una función de provisora de servicios sociales, que influye sobre la división de tareas en las familias y sobre las posibilidades de producción de nuevos roles familiares. Sería muy interesante poder contar con datos similares para otros países del sur de Europa o que poseen un régimen de bienestar conservador, para poder dilucidar hasta qué punto el nivel de desarrollo de los servicios sociales es responsable parcial de las posibilidades de las mujeres para acceder al mercado de trabajo.

En cuanto al desarrollo de los servicios sociales personales en España, el retraso comparativo obedece a razones históricas, pero también culturales. D e hecho, la escasa tradición asociacionista y en la reclamación de prestaciones sociales al Estado puede explicar la ausencia de una presión mayor para el crecimiento de dichos servicios públicos. En países como Francia, Bélgica y 
H olanda que comparten con España el carácter profesionalista de su sistema de garantía de ingresos, las prestaciones familiares están más desarrolladas muy probablemente porque existen asociaciones activas y con gran número de miembros, asociaciones que, además, cuentan con mecanismos institucionales que les permiten participar y presionar en la formulación de políticas sociales para la familia (I glesias de U ssel y M eil, 1994).

Por otra parte, muchos ciudadanos no quieren tener que reconocer públicamente que dejan a sus padres e hijos en manos de instituciones públicas, a lo que ayuda una percepción de los servicios sociales con regusto a beneficencia, es decir, a instituciones de mala calidad en las que existe el hacinamiento, en servicios para pobres, para los que no tienen otra posibilidad. Además, existe un fuerte control social y profesional (médicos, psicólogos) que defiende la idea de que los bebés y los niños pequeños deben pasar el mayor tiempo posible con sus madres, idea reforzada por la mentalidad tradicional. Tal como indica Valiente (1995c: 9), se asume que las mujeres tienen un instinto natural del que carecen los hombres, por lo que, aunque deban ayudar en el cuidado de los hijos, corresponde sobre todo a las mujeres el ocuparse de ellos. Esto está relacionado con el uso de las guarderías privadas o públicas, de forma que se consideran la última opción cuando la mujer trabaja y no queda más remedio, prefiriendo ponerlos al cuidado de abuelos o de niñeras contratadas para ello cuando los recursos económicos lo permiten. Con respecto a los ancianos, muchas personas consideran que es un deber de los hijos hacia los padres el asistirlos en sus últimos años.

Todo esto tiene la consecuencia, nada deseable, de que el Estado se ve eximido, 0 al menos poco presionado, para solucionar activamente una larga y variopinta lista de problemas sociales: la atención a los ancianos, minusválidos y enfermos mentales, el cuidado de los más jóvenes, una mayor creación de empleo, la vivienda, etc. Aún así es de destacar que la situación está comenzando a cambiar. H oy día los ciudadanos españoles se muestran partidarios en su mayoría de que se incrementen prestaciones familiares tales como medidas fiscales, equipamientos, condiciones laborales y transferencias y se trata, además, de demandas que se han ido fortal eciendo en los últimos años. Esta situación de escasez de oferta unida a una demanda creciente hace que el hecho de que hasta la fecha no se haya producido un debate público sobre el rumbo que debe tomar la política social para la familia se haga cada vez más preocupante (Iglesias y M eil, 1994).

\section{Sistema de garantía de ingresos y mercado de trabajo ${ }^{6}$}

El sistema de garantía de ingresos en España, contrariamente a lo que ocurre en el ámbito de la asistencia sanitaria, presenta hoy día todas las característi-

6. En esta sección sigo de cerca la argumentación de Esping-Andersen (1995). También utilizo los análisis de Pérez D íaz y Rodríguez (1994) y de Bentolila y D olado (1994) sobre la evolución del mercado de trabajo en España. 
cas de un modelo profesionalista propio del régimen de bienestar conservador. En primer lugar, los derechos a recibir prestaciones públicas se derivan de la condición de trabajador y no de la de ciudadano. Consecuentemente, el sistema está fragmentado por categorías profesionales. Aunque la tendencia ha sido, ya desde hace décadas, a la convergencia con el régimen general, aún existen diferencias en la regulación de los distintos regímenes ${ }^{7}$. El grado de fragmentación no es muy alto y es parecido al de los países del centro de Europa. Francia, Italia y G recia poseen un sistema mucho más fragmentado que el español, mientras que en Portugal las divisiones son menores. En segundo lugar, los beneficios van unidos a los niveles salariales. En España, al igual que ocurre en el resto de los países del sur de Europa, el nivel de sustitución de rentas es muy elevado en términos comparativos, corroborando el argumento de Esping-Andersen (1990 y 1995) que defiende que lo que se está sustituyendo es un salario familiar y no individual. Las pensiones contributivas de jubilación de los trabajadores que han seguido una carrera profesional completa y sin interrupciones suponen entre el $89 \%$ y el 107\% del salario medio neto en los países del sur de Europa, mientras que los porcentajes decrecen en los países centroeuropeos y no llegan a alcanzar el 50\% en Gran Bretaña, I rlanda y D inamarca, países que poseen un régimen de bienestar más cercano al socializado. La «generosa» protección del sector formal del mercado de trabajo contrasta con una atención mucho más débil a los sectores más informales o irregulares, en los que se encuentran trabajadores que no cumplen las condiciones para tener derecho a una pensión contributiva (Ferrara, 1995).

En España, tal como ha ocurrido en otros casos nacionales que disfrutan de regímenes del bienestar conservadores, se han ido introduciendo medidas de política social de corte más universalista, dirigidas a los ciudadanos sin acceso a la Seguridad Social: pensiones no contributivas, prestaciones familiares, subsidios de desempleo, transferencias todas ellas que se financian en su mayor parte a través de impuestos y que van dirigidas a los niveles de renta más bajos. Estos desarrollos han incidido en una participación creciente de los Estados en la financiación de las prestaciones para hacer frente al incremento de los beneficios, al aumento en el número de perceptores (debido al envejecimiento de la población, al aumento de la esperanza de vida, pero también al volumen de las jubilaciones anticipadas) y a la inclusión de nuevos grupos bajo el manto protector. Para el caso concreto de nuestro país, la universalización de las prestaciones para los mayores de 65 años prácticamente se ha logrado, pero la protección de las personas en edad activa es menor en términos comparativos con otros países (Comisión Europea, 1994). D e hecho, la distribución del gasto ha variado mucho: mientras que en 1967 las pensiones suponían la mitad del gasto en todos los beneficios económicos, en 1986 habían alcanzado casi el

7. Además del régimen general para trabajadores dependientes, existen en España regímenes especiales para los trabajadores de la minería del carbón, los trabajadores del mar, los autónomos, el servicio doméstico y los trabajadores agrícolas. Los funcionarios públicos están cubiertos por régimen independiente. 
89\% (Velarde, 1990: 131). Esto quiere decir que se ha priorizado claramente un área de la política social, la del mantenimiento de rentas de las personas de edad, a costa de otras áreas, al menos, de las encargadas de la protección de las personas en edad activa. Pero cuando el desempleo aumenta, los matrimonios son cada vez menos estables y los hogares unipersonales más frecuentes, esto puede suponer un serio problema. Por el momento, tal como se ha visto en la sección anterior, las familias están encargándose de servir de apoyo para sobrellevar estas situaciones, pero no sabemos cuánto tiempo estarán dispuestas a seguir haciéndolo. Al contrario, todo parece indicar que las mujeres desean trabajar y lo hacen en proporciones cada vez mayores, así como que no están dispuestas a interrumpir su carrera profesional para dedicarse al cuidado de los hijos, lo cual redunda en tasas de fecundidad cada vez más bajas.

Por otra parte, el hecho de que los trabajadores deban permanecer en sus puestos durante períodos prolongados de tiempo para acceder al sistema contributivo hace que los sistemas profesionalistas hayan aparecido históricamente asociados con mercados de trabajo que ofrecen altas dosis de seguridad en el empleo. Esta combinación funcionó bien mientras se mantuvieron tasas de inflación y de desempleo moderadas. Sin embargo, la respuesta de los regímenes de bienestar conservadores a las consecuencias de las crisis económicas ha consistido en la reducción de la fuerza de trabajo mediante jubilaciones anticipadas y otras medidas similares. D esde luego, esta estrategia parece haber ido unida, en todos los países con regímenes de bienestar conservadores, al mantenimiento del sistema de bienestar profesionalista, que no se ha intentado modificar en ninguno de ellos. Se trata de una estrategia que está basada en dar salida a los excedentes del mercado de trabajo mediante medidas de protección social, a la vez que se reducen drásticamente las nuevas incorporaciones.

Esto ha parecido una medida adecuada a los poderes públicos porque desde ellos se ha supuesto erróneamente que el incremento de productividad que debe acompañarla puede compensar el gasto en transferencias sociales y que, además, el superávit de trabajadores no especializados es transitorio y el problema se solucionará una vez que el período de reconversión industrial llegue a su término (Esping-Andersen, 1995). La pertinencia de dicha medida queda en entredicho cuando ya se ha constatado que los resultados de esta política han conducido a una situación en la que la razón entre contribuyentes y dependientes cada vez es más desfavorable, es decir, que ha deteriorado la salud financiera del sistema de aseguramiento social. Además, los al tos costes laborales fijos tienen una incidencia negativa sobre la creación de empleo e inducen el crecimiento de los sectores informales o del autoempleo, con la consecuencia aña dida de que se pueden mantener o incluso fortalecer los privilegios del sector formal porque las empresas son capaces de flexibilizar su plantilla a través de contratos temporales y/o sumergiendo parte de su actividad productiva. Por su parte, el mantenimiento de altas tasas de desempleo también conlleva el surgimiento de tensiones financieras, debido al considerable coste que supone la cobertura de esta contingencia. Asimismo, el elevado gasto en protección por 
desempleo incide negativamente sobre la creación de puestos de trabajo porque detrae recursos que podrían emplearse en la aplicación de políticas activas de mercado de trabajo.

La atenuación de las rigideces del mercado de trabajo puede constituir un mecanismo que facilite la creación de empleo y con ello favorecer la inserción laboral de jóvenes y mujeres. La flexibilización del mercado de trabajo para adecuarlo a la nueva situación económica se ha hecho de forma tímida y lenta en España, y se ha producido a grandes rasgos en dos etapas. La primera, iniciada en 1984, incentivó la creación de puestos de trabajo con contratos temporales, de forma que en el momento presente un tercio de todos los empleos son de este tipo. La segunda, realizada entre diciembre de 1993 y mayo de 1994, supuso a grandes rasgos la promoción de la creación de empleo a través del establecimiento de exenciones de impuestos y contributivas para los empresarios que contrataran a jóvenes, desempleados de larga duración, mayores de 45 años y minusválidos; la incentivación de los contratos de aprendizaje y en prácticas, así como de los empleos a tiempo parcial, y la reducción de las barreras para cierto tipo de despidos.

H oy día resulta difícil defender que todavía nos encontramos ante un mercado de trabajo poco flexible, máxime cuando los datos extraídos de las declaraciones a $\mathrm{H}$ acienda de los empresarios indican que más de la cuarta parte de los asalariados ganaban menos del salario mínimo en 1992 (C arabaña, 1995). Sin embargo, hay que resaltar el hecho de que todas estas medidas de flexibilización han afectado a los nuevos contratos y no a los ya existentes, de forma que han conducido a una segmentación clara del mercado de trabajo entre el sector formal y más protegido, por un lado, y, por otro, los puestos de trabajo temporal, a tiempo parcial, contratos en prácticas y de aprendizaje, etc. y el sector sumergido o informal. Las mujeres españolas han mejorado sus cualificaciones significativamente en los últimos quince años y los niveles de educación se han equiparado entre ambos géneros, pero el acceso al mercado de trabajo, al igual que les ha ocurrido a los jóvenes, se ha visto mediatizado por las nuevas condiciones de flexibilización parcial, de forma que los puestos a los que han podido acceder son en muchos casos empleos peor pagados y mucho más precarios que los del sector formal. Esto afecta claramente a las oportunidades que tienen las mujeres para alcanzar una independencia económica, pues dicha independencia es bastante frágil cuando una persona sale y entra del mercado continuamente y no sabe cuantas veces se podrá seguir manteniendo esa alternancia.

Además de las diferencias por género y edad que se pueden observar en el mercado entre el núcleo más protegido, predominantemente masculino y los sectores temporal e informal, nutridos en mayor proporción por jóvenes y mujeres, tal como indica Garrido M edina (1992), la evolución del mercado de trabajo también ha supuesto el surgimiento de segmentaciones claras dentro del grupo de mujeres que trabajan fuera del hogar. La primera de ellas, y quizás la más importante, es una segmentación por edad, entre las mujeres jóvenes que han podido acceder a la formación y que, educadas o no, han tenido más 
oportunidades de inserción laboral durante los ciclos expansivos de la economía y las mujeres de más edad que no han contado con estas oportunidades. Una segunda diferencia se ha establecido entre las mujeres con altos niveles de educación, que se han incorporado sobre todo al sector público pero también han logrado insertarse en la empresa privada, y las que carecen de formación o la poseen en muy escasa medida, para las que las oportunidades de encontrar trabajo son raras.

Cuando para disfrutar de una pensión de jubilación la estabilidad en el empleo se hace necesaria, lo lógico es no correr riesgos y que aquella persona que tenga un trabajo fijo dentro de la unidad familiar cuide de él. Puesto que son los hombres los que ocupan ya y los que acceden con más facilidad a los puestos de trabajo seguros, en caso de que haya que escoger siempre se tenderá a priorizar dicho empleo sobre el de la mujer, habitualmente menos seguro y peor pagado. 0 tra consecuencia es que, incluso en el caso de que las mujeresinterrumpan su vida laboral y luego tengan la suerte de poder reincorporarse a ella, las pensiones disfrutadas por mujeres son siempre muy inferiores a las de los hombres. En resumidas cuentas, todo esto redunda en menores posibilidades de alcanzar la independencia económica durante la edad activa y también tras la jubilación, y, con ello, escasas oportunidades para que se modifiquen los roles familiares. En todo caso, parece que en muchas circunstancias resulta más rentable que las mujeres realicen trabajos en el propio hogar: actividades más o menos sumergidas, que les permitan compatibilizar mejor las tareas domésticas con una contribución al salario familiar.

\section{Conclusiones}

Si se combinan la existencia de parcas prestaciones familiares y de escasez de servicios para el cuidado de los niños, ancianos y minusválidos y otros servicios para las familias, no es de extrañar que las tasas de actividad femenina sean más bajas en los países que han construido un régimen de bienestar conservador, y que la producción de nuevos roles familiares se vea dificultada.

Por otra parte, las relaciones mutuas entre un sistema de garantía de ingresos de corte profesionalista y un mercado de trabajo segmentado en un sector formal y fuertemente regulado, otro de empleo precario y otro informal tienen consecuencias claras sobre la incorporación de las mujeres a la vida laboral, y con ello sobre las posibilidades de modificar su rol familiar tradicional. Ambas configuraciones - la del estado del bienestar y la del mercado de trabajoparecen generar un círculo vicioso de difícil salida.

Las medidas de control de costes que se han propuesto para asegurar el futuro del sistema, tales como cambios en la indiciación de las pensiones, en los períodos requeridos para acceder a las prestaciones, el retraso de la edad de jubilación, la mayor correspondencia entre contribuciones, etc. pueden hacer viables desde el punto de vista financiero los sistemas de mantenimiento de rentas, pero no suponen la solución definitiva. Lo mismo ocurre con la utilización de subsidios o exenciones fiscales para fomentar el empleo. En 
definitiva se trata de medidas parciales, de parches que permiten paliar los problemas.

U na solución parecida a la adoptada por los países escandinavos no parece factible, dadas las dificultades de financiación por las que están atravesando todos los estados del bienestar, que hace muy difícil la posibilidad de una fuerte expansión de los programas sociales. Además, tal como se ha señalado, estos regímenes de bienestar también llevan apareada una segmentación del mercado de trabajo, en este caso una segmentación por género. La solución emprendida por los regímenes liberales, como por ejemplo el de Estados U nidos, consistente en favorecer la flexibilidad de los salarios, incrementa la desigualdad social y parece, desde luego, difícilmente exportable al entorno cultural del centro y sur de Europa.

Resulta, pues, bastante arduo buscar modelos a seguir en el exterior. Pero en estos momentos en que nos estamos planteando la reforma del estado del bie nestar, sobre todo en el área de la garantía de ingresos, a la vez que buscamos afanosamente los medios que conduzcan a una mayor creación de empleo, cabe recordar y tener bien presente cuáles son las consecuencias que conlleva la construcción de un régimen de bienestar u otro y qué modelo deseamos para nuestra sociedad en el futuro. ¿D eseamos vivir en una sociedad dualizada, con trabajadores de primera y de segunda clase? ¿Q ueremos un sistema que siga distribuyendo el trabajo de forma que sean los hombres los que se encarguen de ganar el salario familiar y las mujeres de las labores del hogar? ¿C ontinuaremos ocupándonos concienzudamente de que las mujeres se formen igual de bien que los hombres para que luego muchas de ellas cuelguen su título académico o su acreditación profesional en la cocina? ¿Seguiremos permitiendo que la incorporación de las mujeres al mercado de trabajo tenga que ir unida a tasas de fecundidad decrecientes? El debate está abriéndose camino y vale la pena que se diriman las consecuencias de las posibles opciones con sumo cuidado.

\section{Bibliografía}

Bento LILA, S.; D OLAD O, J. (1994). «Labour Flexibility and Wages: Lessons from Spain». Economic Policy, 18, p. 55-99.

Boletín CIRES (mayo de 1994). Las demandas sociales de bienestar. M adrid: CIRES. C ARABAÑ A, J. (1995). «Un mercado de trabajo bien flexible». Claves de razón práctica,

CASAD O , D. (dir.) (1994). «Acción social y servicios sociales». En V Informe sociológico sobre la situación social en España. M adrid: Fundación FO ESSA.

- (1992). «Política española concerniente a la discapacidad». En Política social y estado del bienestar. M adrid: M inisterio de Asuntos Sociales.

Consejo ECon ómico y So cial (1995). España 1994. Economía, trabajo y sociedad. M emoria sobre la situación socioeconómica y laboral. M adrid: CES.

CIRES (1994). La realidad social en España 1992-1993. Barcelona: Ediciones B.

COMISIÓN EUROPEA (1994). La protección social en Europa. Luxemburgo: O ficina de Publicaciones $\mathrm{O}$ ficiales de las $\mathrm{C}$ omunidades Europeas. 
Espin G-Andersen (1990). The Three Worlds of Welfare Capitalism. O xford: Polity Press.

- (1995). «W elfare States without Work: The Impasse of Labor Shedding and Familialism in Continental European Social Policy». Estudios/Working Papers, 71. $M$ adrid: Instituto Juan $M$ arch de Estudios e Investigaciones.

FerRera, M . (1995). «T he Southern Welfare States in Social Europe». Journal of European Social Policy. En prensa.

FUNDACIÓN FOESSA (1994). V Informe sociológico sobre la situación social en España. $M$ adrid: FOESSA.

GaRRID O M EDINA, L. (1992). Las dos biografías de la mujer en España. M adrid: M inisterio de Asuntos Sociales e Instituto de la M ujer.

- (1993). «La familia estatal: el control fiscal de la natalidad». En GARRIDo M edin A, L.; GIL Calvo, E. (ed.). Estrategias familiares. M adrid: Alianza.

G UILLEM ARD, A.M . (1993). «Perspectivas europeas sobre las políticas de la vejez». En M OREN O, Luis (ed.). Intercambio social y desarrollo del bienestar. M adrid: CSIC.

I GLESIAS DE U SSEL, J.; M EIL, G . (1994). «La política familiar desde la transición». En $\checkmark$ Informe sociológico sobre la situación social en España. M adrid: FO ESSA.

In STITUTO N ACIONAL DE ESTADístICA (1986). Encuesta sobre discapacidades deficiencias y minusvalías. Madrid: IN E.

Fernández M Éndez de And RÉs, F. (dir.) (1987). Actividad laboral de la mujer en relación a la fecundidad. M adrid: M inisterio de Cultura e Instituto de la M ujer.

M In ISTERIO de Asuntos So Ciales (1989). Prestaciones y servicios para personas con minusvalía en los países de la CE.

- (1993). Indicadores de protección social. Servicios sociales y programas de igualdad durante el período 1982-1992. M adrid: M inisterio de Asuntos Sociales.

M und AY, B. (ed.) (1993). European Social Services. Universidad de Kent: European Institute of Social Services.

N avarro, M . (1994). «Relaciones de parentesco». En Cam po, S. del (ed.). Tendencias Sociales en España (1960-1990). Bilbao: Fundación BBV.

Pérez D íaz y Rod Ríguez (1994). «O pciones inerciales. Políticas y prácticas de recursos humanos en España (1959-1993)». ASP Research Papers 2(a).

Kersbergen, K. van (1995). Social Capitalism. Londres: Routledge and Kegan Paul.

RAM os T ORRES, R. (1990). Cronos dividido. U so del tiempo y desigualdad entre hombres y mujeres en España. M adrid: M inisterio de Asuntos Sociales e Instituto de la M ujer.

Rodríguez C ABrero (1994). «La protección social a la familia». En V Informe sociológico sobre la situación social en España. M adrid: Fundación FO ESSA.

V ALIENTE, C. (1995a). «C hildren First: Central Government Child Care Policies in Post-Authoritarian Spain (1975-1994)». En BRAN N EN, Julia; BRIEN, M argaret O. (ed.). Childhood and Parenthood. Universidad de Londres: Instituto de Educación.

- (1995b). «Rejecting the Past: Central Government and Family Policy in PostAuthoritarian Spain (1975-1994)». En H ANTRAIS, Linda; LetABLIER, M arieT hérèse (ed.). The Family in Social Policy and Family Policy. Cross N ational Research Papers, 4 (3), p. 80-96.

- (1995c). «M en's Family Roles in Post-Authoritarian Spain (1975-1995)». Universidad de Gotemburgo. En prensa.

VelARD E (1990). El tercer viraje de la Seguridad Social. M adrid: Instituto de Estudios Económicos. 